ARTICLE OPEN

\title{
Using a smartphone-based self-management platform to support medication adherence and clinical consultation in Parkinson's disease
}

Rashmi Lakshminarayana', Duolao Wang ${ }^{2}$, David Burn ${ }^{3}$, K. Ray Chaudhuri ${ }^{4}$, Clare Galtrey ${ }^{5}$, Natalie Valle Guzman ${ }^{6}$, Bruce Hellman ${ }^{1}$, Ben James ${ }^{1}$, Suvankar Pal ${ }^{7}$, Jon Stamford ${ }^{8}$, Malcolm Steiger ${ }^{9}$, R. W. Stott ${ }^{6}$, James Teo ${ }^{10}$, Roger A. Barker ${ }^{11}$, Emma Wang ${ }^{12}$, Bastiaan R. Bloem ${ }^{13}$, Martijn van der Eijk ${ }^{13}$, Lynn Rochester ${ }^{3}$ and Adrian Williams ${ }^{14}$

The progressive nature of Parkinson's disease, its complex treatment regimens and the high rates of comorbid conditions make self-management and treatment adherence a challenge. Clinicians have limited face-to-face consultation time with Parkinson's disease patients, making it difficult to comprehensively address non-adherence. Here we share the results from a multi-centre (seven centres) randomised controlled trial conducted in England and Scotland to assess the impact of using a smartphone-based Parkinson's tracker app to promote patient self-management, enhance treatment adherence and quality of clinical consultation. Eligible Parkinson's disease patients were randomised using a 1:1 ratio according to a computer-generated random sequence, stratified by centre and using blocks of variable size, to intervention Parkinson's Tracker App or control (Treatment as Usual). Primary outcome was the self-reported score of adherence to treatment (Morisky medication adherence scale -8 ) at 16 weeks. Secondary outcomes were Quality of Life (Parkinson's disease questionnaire -39), quality of consultation for Parkinson's disease patients (Patient-centred questionnaire for Parkinson's disease), impact on non-motor symptoms (Non-motor symptoms questionnaire), depression and anxiety (Hospital anxiety and depression scale) and beliefs about medication (Beliefs about Medication Questionnaire) at 16 weeks. Primary and secondary endpoints were analysed using a generalised linear model with treatment as the fixed effect and baseline measurement as the covariate. 158 patients completed the study (Parkinson's tracker $\mathrm{app}=68$ and TAU =90). At 16 weeks Parkinson's tracker app significantly improved adherence, compared to treatment as usual (mean difference: $0.39,95 \% \mathrm{Cl} 0.04-0.74 ; p=0.0304$ ) with no confounding effects of gender, number of comorbidities and age. Among secondary outcomes, Parkinson's tracker app significantly improved patients' perception of quality of consultation $(0.15$, $95 \% \mathrm{Cl} 0.03$ to $0.27 ; p=0.0110)$. The change in non-motor symptoms was $-0.82(95 \% \mathrm{Cl}-1.75$ to $0.10 ; p=0.0822)$. $72 \%$ of participants in the Parkinson's tracker app group continued to use and engage with the application throughout the 16-week trial period. The Parkinson's tracker app can be an effective and novel way of enhancing self-reported medication adherence and quality of clinical consultation by supporting self-management in Parkinson's disease in patients owning smartphones. Further work is recommended to determine whether the benefits of the intervention are maintained beyond the 16 week study period.

npj Parkinson's Disease (2017)3:2 ; doi:10.1038/s41531-016-0003-z

\section{INTRODUCTION}

Parkinson's disease (PD) is the second most common neurodegenerative disorder, after Alzheimer's disease ${ }^{1}$ and affects 6.3 million people worldwide. ${ }^{2}$ It is a disabling condition and has a significant impact on patient's Quality of Life (QoL). ${ }^{3}$ Non-motor symptoms such as depression, anxiety, fatigue and sleep disturbance are frequently overlooked by clinicians ${ }^{4}$ and contribute to significant burden on people with PD and their carers. ${ }^{5}$ Comorbidity is common in PD. Dementia, arthritis, ischaemic heart disease, diabetes and falls are amongst the commonly associated co-morbidities. ${ }^{6}$

Managing motor and non-motor symptoms and the risk of side effects from medications leads to complex treatment regimes. ${ }^{7}$
The number of drugs and the frequency with which they are taken typically increases as the diseases progresses. ${ }^{8,9}$ The addition of medications to manage associated co-morbidities further adds to the complexity of managing dosing schedules.

Patient centred care-" providing care that is respectful of and responsive to individual patient preferences, needs and values, and ensuring that patient values guide all clinical decisions" ${ }^{\prime 10}$-is a core aspect of quality of care and increases treatment adherence among chronically ill patients and job satisfaction among health professionals. ${ }^{11}$ Self-management support and shared decisionmaking have been identified as two promising ways to support and empower PD patients. ${ }^{11}$

\footnotetext{
${ }^{1}$ uMotif Ltd, London, UK; ${ }^{2}$ Liverpool School of Tropical Medicine, Liverpool, UK; ${ }^{3}$ Newcastle-upon-Tyne Hospitals NHS Foundation Trust, Newcastle Upon Tyne, UK; ${ }^{4}$ King's College Hospital NHS Foundation Trust, London, UK; ${ }^{5}$ St George's Healthcare Trust, London, UK; ${ }^{6}$ John van Geest Centre for Brain Repair, Cambridge, UK; ${ }^{7}$ NHS Forth Valley, Scotland, UK; ${ }^{8}$ Cure Parkinson's Trust, London, UK; ${ }^{9}$ The Walton Centre NHS Foundation Trust, Liverpool, UK; ${ }^{10}$ King's College Hospital NHS Foundation Trust, London, UK; ${ }^{11}$ John van Geest

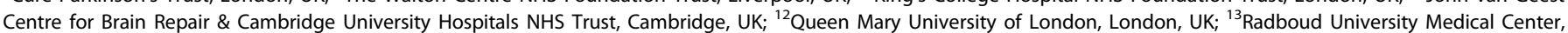
Nijmegen, The Netherlands and ${ }^{14}$ University Hospitals Birmingham NHS Foundation Trust, Birmingham, UK Correspondence: Rashmi Lakshminarayana (contact@umotif.com)
} 
Self-management support refers to increasing patient participation, collaborative goal setting, treatment planning and assisting patients to gain control over their lives. ${ }^{12}$ It can teach PD patients how to better utilise healthcare resources and how to form more effective partnerships with their care providers. ${ }^{11}$

The reported prevalence of non-adherence to prescribed therapy in PD varies from 0 to $60-70 \%{ }^{1}$ Medication nonadherence can be unintentional or intentional. ${ }^{13}$ Unintentional nonadherence involves intending to take a medication as instructed but failing to do so for some reason (e.g. forgetfulness, carelessness) and is influenced by patient characteristics, treatment factors, and patient-provider issues. ${ }^{14}$ Intentional nonadherence involves making a reasoned decision not to take a medication as instructed based on perceptions, feelings or beliefs. It reflects a rational decisionmaking process by the patient whereby the benefits of treatment are weighed against any adverse effects of the treatment. ${ }^{14}$ The direct and indirect costs of nonadherence in the USA is between $\$ 100-\$ 300$ billion/year ${ }^{15}$ and over $£ 930$ million/year in England. ${ }^{16}$ Nonadherence is linked to poor QoL, increased hospitalisation admissions and premature mortality. ${ }^{17,18}$

Delivering self-management support digitally, including support for understanding and managing treatment has shown improvements health outcomes and processes of care in other chronic conditions. ${ }^{19}$ The SMART-PD trial aimed to assess outcomes of a patient centred smartphone and Internet assisted selfmanagement and treatment adherence tool, the Parkinson's Tracker App (PTA), to manage PD. Two versions of the PTA were assessed in a previous randomised pilot study in collaboration with the Cure Parkinson's Trust UK. ${ }^{20}$ Patient feedback on design, features and usability from this study was used to update the PTA for the current trial.

Our primary objective was to assess if patients with PD who use a PTA for 16 weeks in addition to TAU (treatment as usual) show improved self-reported medication adherence. Our secondary objectives were to investigate whether patients who receive the PTA and those who receive TAU differ in terms of QoL, quality of clinical consultation and symptom control.

\section{RESULTS}

We invited 737 patients to participate. Of the 522 (70.8\%) who responded, 277 (53\%) did not meet the inclusion criteria, 65 (12.4\%) declined to participate and 180 (34.5\%) could not take part due to other reasons including not having devices with iPhone/iPad or Android operating systems or being unable to make it to the OutPatient appointment. Figure 1 highlights the trial CONSORT flow.

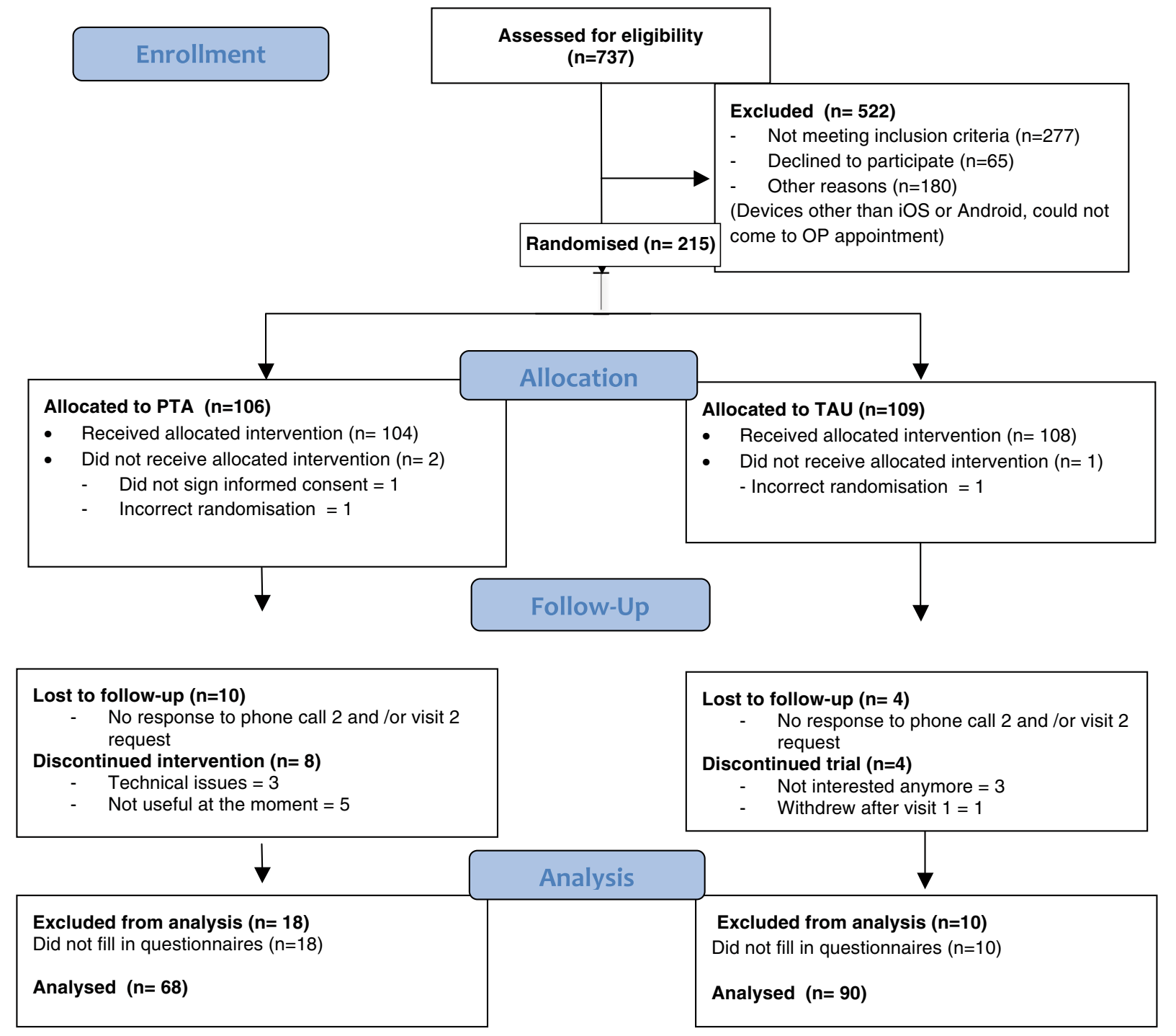

Fig. 1 Trial CONSORT patient flow diagram 


\begin{tabular}{|c|c|c|c|}
\hline Variable & $\begin{array}{l}\text { PTA }^{\mathrm{a}} \\
(N=94) \\
\text { Mean (SD) }\end{array}$ & $\begin{array}{l}\text { TAU }^{\mathrm{b}} \\
(N=107) \\
\text { Mean (SD) }\end{array}$ & $\begin{array}{l}\text { All } \\
(N=201)\end{array}$ \\
\hline Age at screening (year) & $59.86(9.13)$ & $60.71(10.26)$ & $60.31(9.73)$ \\
\hline \multicolumn{4}{|l|}{ Gender } \\
\hline Number of comorbidities & $1.39(1.66)$ & $1.32(1.59)$ & $1.35(1.62)$ \\
\hline Parkinson's disease duration (years) & $5.47(4.18)$ & $5.47(4.89)$ & $5.47(4.56)$ \\
\hline Morisky Medication Adherence Scale (MMAS- $8^{\mathrm{C}}$ ) & $6.03(1.57)$ & $5.82(1.48)$ & $5.92(1.52)$ \\
\hline Quality of life (PDQ-39) & $154.53(27.98)$ & $151.43(27.70)$ & $152.88(27.81)$ \\
\hline Patient-Centered Questionnaire for Parkinson's Disease (PCQ-PD) & $1.91(0.53)$ & $1.93(0.51)$ & $1.92(0.52)$ \\
\hline Beliefs about Medication Questionnaire (BMQ) & $51.83(9.48)$ & $51.93(8.09)$ & $51.88(8.74)$ \\
\hline Number who need help with their medication $(n)$ & 19 & 27 & 46 \\
\hline \multicolumn{4}{|c|}{$\begin{array}{l}\text { a PTA Parkinson's tracker app } \\
\text { b Treatment as usual } \\
\text { c Use of the OMMAS is protected by US copyright laws. Permission for use is required. A license agreement is available from: Donald E. Morisky, MMAS } \\
\text { Research (MORISKY) } 14725 \text { NE 20th St. Bellevue, WA 98007; dmorisky@gmail.com }\end{array}$} \\
\hline
\end{tabular}

The remaining 215 patients were randomly assigned to PTA $(n=106)$ and control $(n=109)$. Of these, 10 patients $(9.4 \%)$ and 4 (3.7\%) were lost to follow-up in PTA and TAU arm, respectively. Of those who completed the trial, we analysed data from 68 (79\%) and $90(90 \%)$ in the PTA and TAU arms, respectively. We closed recruitment due to funding restrictions.

Baseline data were comparable between the two groups (Table 1). Participants took medications for 42 co-morbid conditions in addition to PD (Table 2). Participants took between 1 to 5 types of medications to manage PD. The majority of participants were White $(n=206)$ with a small number being Asian/Asian British $(n=6)$ and Black/Black British $(n=2)$.

\section{Primary outcome}

Participants in the PTA group $(n=68)$ showed an improvement in MMAS-8 $8^{21,22,23}$ score of 0.39 points over TAU group in Intent-toTreat analysis (Table 3) (95\% Cl 0.04 to $0.74 ; p=0.0304$ ), translating to better self-reported adherence to medication. These findings were replicated in Per Protocol population $(0.41,95 \% \mathrm{Cl} 0.06$ to 0.76 ;

$p=0.0227)$. This translated into a $6.6 \%$ reduction in low adherence category in the PTA group compared to $1.4 \%$ reduction in the TAU group (Fig. 2).

When the ANCOVA analysis was adjusted for the covariates gender, number of comorbidities and age, the effect of treatment remained statistically significant $(0.38,95 \% \mathrm{Cl} 0.03$ to 0.73 ; $p=0.0301$ (Table 3), illustrating that the result of improvement is robust even after controlling for the three covariates on the analysis.

Subgroup analyses with interaction testing were performed to determine whether the improvement in the primary end point was consistent across four important subgroups (Table 4). No significant interactions were observed. However, results revealed that those older than 61, females and those with greater than one comorbidity tended to benefit more from PTA than their counterparts.
Secondary outcomes

Among the secondary outcomes (Table 5) the PCQ-PD improved in the PTA group $(p=0.0110)$. Quality of PD care was assessed in 6 dimensions in PCQ-PD (i.e. information, collaboration, accessibility, empathy, patient involvement and emotional support). Statistically significant improvements were seen in patient perception of collaboration and patient involvement in decision-making subscales.

The difference between the PTA and TAU groups for the secondary endpoint of Non-Motor Symptom Questionnaire was $-0.82(p=0.0822)$ and for the QoL questionnaire (PDQ-39) was $-0.22(p=0.9102)$. Results for individual subdomains of PDQ39 are presented in Supplementary Table 1.

There were no statistically significant differences between the groups with regards to other secondary outcomes. These findings were consistent with per protocol analysis.

\section{Analysis of PTA use}

Seventy-two per cent of participants in the PTA group continued to use and engage with the application throughout the 112-day trial period (Table 6). They used the app almost every other day on average, with some people using the app every day (Table 7).

We undertook further analysis of PTA usage in the PTA group in March 2016, after the end of the study in June 2015. The table below (Table 8 ) highlights that $29 \%$ of participants continued to use the app for over 6 months after the trial had ended.

\section{DISCUSSION}

Compared to TAU, participants using the PTA for 16 weeks reported statistically significant improvements in short-term selfreported medication adherence and subjective quality of clinical consultation. These findings suggest that the PTA can be useful in improving outcomes and processes of care in people with PD, similar to results seen in other chronic conditions such as diabetes and asthma. ${ }^{19}$ Given that typical clinical follow-up of patients with PD by clinical care teams in the UK occurs approximately every 6 months (by consultants or nurse specialists), this finding 
decision-making. ${ }^{11}$ Other studies have found that PD patients who perceived higher involvement in their care were more satisfied with the consultation and intended to be more compliant with treatment. ${ }^{29}$ Those in the PTA group perceived the service as more patient-centred as measured on the PCQ-PD $(p=0.01)$. This could be linked to clinical teams inviting patients to use the app, personalising the app set-up and jointly reviewing progress using the data collected by patients at the 16-week follow-up.

User retention is a recognised challenge for all Smartphone apps, with less than one in seven people opening apps beyond a day after download. ${ }^{30}$ The higher level of user retention during the SMART-PD trial can be linked to factors including the simplicity and design of the app's user interface and user experience; that the app is perceived as given by participant's clinical team and thereby acting as an extension of their recommended care; and the direct benefit of understanding their health better as reported by PTA users.

The trial included only those who already had smartphones or tablet devices, thereby excluding those who did not own such a device. Smartphone penetration in the UK is estimated at $66 \%$, ranging from $88 \%$ in those aged $25-34$ to $49 \%$ in those aged $55-64$ and $17 \%$ in those over $65 .{ }^{31}$ These later groups are the most

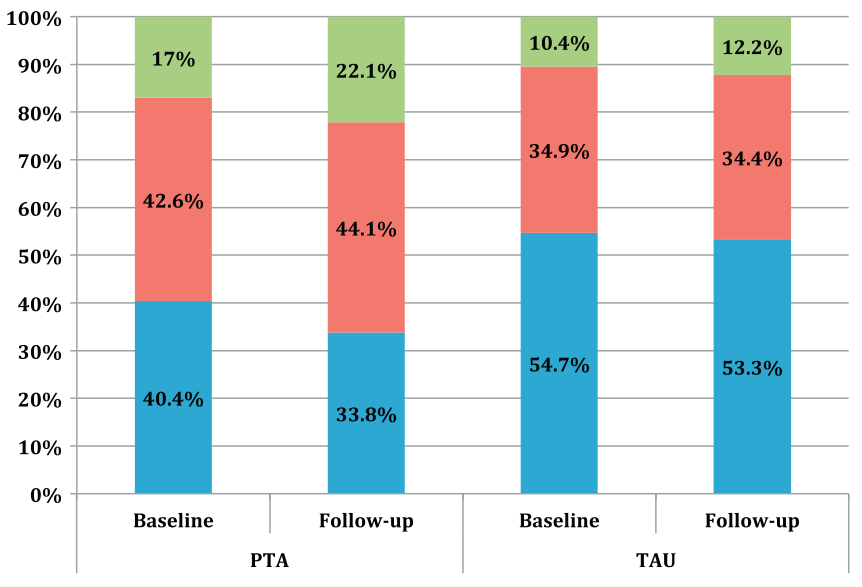

Low adherence Medium adherence High adherence

Fig. 2 Change in adherence categories: PTA and TAU represented in the study group. The study app was available on iOS (Apple operating system) and Android (Google operating system). These were the major operating systems during the study period (August 2014-June 2015) with $42.33 \%$ being iOS users and $47.95 \%$ being Android users. We estimate that users of other operating systems (i.e. $9.72 \%$ of total smartphone and tablet device users during the study period used Windows and Blackberry $)^{32}$ could not take part, further contributing to the reported response rate. As the smartphone market penetration increases, especially in those over 65 , it would be anticipated that more patients would be able to use the study app. It should be noted that a mean age of 60 in the PTA group was not a barrier for interaction with the PTA, allaying fears that technology-based interventions are not appropriate for an older age group.

Our results also clearly highlight the difficulty of studying adherence using a pragmatic design. Research into recruitment rates for randomised controlled trials $(\mathrm{RCTs})^{33}$ highlight the challenges of both recruiting patients and of retentaining recruited patients despite implementing various retention strategies along with letters. Since we did not use any other methods of retention, this could explain the study retention rate we found in the studythere was a higher than expected loss to follow-up and survey completion rates $(64 \%$ in PTA arm and $83 \%$ in the TAU arm completed as Per Protocol). We relied on the use of online questionnaires that patients completed at home, rather than a standard paper-based questionnaire completed at the clinic during study visits, which likely led to the rate of drop-out. However, questionnaires delivered and completed by the patient at home have the benefit of reduced recall bias and in-situ data collection. It is possible that follow-up drop-outs preferentially affect those with poor self-reported medication adherence, the difference in medications adherence between study arms were maintained in both intention-to-treat and per-protocol analysis. The higher study dropout rate in the PTA group is likely due to the participants needing to perform an additional trial task of using the PTA compared to the TAU group. Additionally, the PTA may not have met all participants' needs (for example: some of the self-monitoring measures chosen by the trial team may not be applicable to some participants), which could have contributed to the dropouts.

The MMAS- 8 was developed to manage hypertension ${ }^{20}$ and the MMAS-4 has been used in PD studies. ${ }^{34} \mathrm{~A}$ 2-point improvement on the MMAS-8 scores was found to link to clinically significant improvement in hypertension. ${ }^{35}$ However, there are no similar measures of the MMAS-8 in PD. Some studies investigating

Table 4. Comparison of age at screening, gender, number of co-morbidities and duration of Parkinson's disease between PTA and TAU groups

\begin{tabular}{|c|c|c|c|c|c|}
\hline Subgroup variable & Category & \multicolumn{2}{|l|}{$n$, mean (SD) } & \multicolumn{2}{|l|}{ GLM analysis } \\
\hline \multicolumn{6}{|c|}{ Age at screening (year) } \\
\hline & $\leq 61$ & $33,6.08(1.49)$ & $45,5.69(1.53)$ & $0.08(-0.44,0.59)$ & 0.7752 \\
\hline & $>61$ & $35,6.51(1.54)$ & $45,5.79(1.54)$ & $0.70(0.23,1.17)$ & 0.0036 \\
\hline \multicolumn{6}{|l|}{ Gender } \\
\hline \multicolumn{6}{|c|}{ Number of comorbidities } \\
\hline & $\leq 1$ & $43,6.13(1.64)$ & $59,5.94(1.45)$ & $0.21(-0.18,0.60)$ & 0.2892 \\
\hline & $>1$ & $25,6.59(1.26)$ & $31,5.37(1.63)$ & $0.84(0.15,1.54)$ & 0.0167 \\
\hline \multicolumn{6}{|c|}{ Parkinson's disease duration (year) } \\
\hline & $\leq 4$ & $37,6.56(1.30)$ & $52,5.88(1.55)$ & $0.47(-0.03,0.96)$ & 0.0630 \\
\hline
\end{tabular}


Table 5. Difference in mean scores on secondary outcomes between PTA and TAU groups at 16 weeks (intention-to-treat population)

\begin{tabular}{|c|c|c|c|c|}
\hline Variable & \multicolumn{2}{|l|}{$n$, mean (SD) } & \multicolumn{2}{|l|}{ GLM analysis } \\
\hline QoL (PDQQoL-39) & 68,155.47 (28.16) & $89,150.75$ (25.62) & $-0.22(-3.95,3.52)$ & 0.9102 \\
\hline Patient-Centred Questionnaire for Parkinson's Disease (PCQ-PD) & $68,2.03(0.48)$ & $89,1.85(0.54)$ & $0.15(0.03,0.27)$ & 0.0110 \\
\hline Non-Motor Symptoms Questionnaire (NMSQuest) & $68,9.82(5.68)$ & $89,10.66(4.89)$ & $-0.82(-1.75,0.10)$ & 0.0822 \\
\hline Hospital Anxiety Rating Scale (HADSa) & $68,6.03(4.15)$ & $89,6.31(4.21)$ & $0.30(-0.42,1.01)$ & 0.4136 \\
\hline
\end{tabular}

\begin{tabular}{|lrrrr|}
\hline $\begin{array}{l}\text { Table 6. } \\
(n=68)\end{array}$ & PTA user retention over trial period (August 2014-June 2015) \\
\hline & \multicolumn{5}{l}{ Proportion of trial complete } \\
\cline { 2 - 6 } & $25 \%$ & $50 \%$ & $75 \%$ & $100 \%$ \\
\hline Number of days used & 28 & 56 & 84 & 112 \\
Number of patients using app & 56 & 56 & 53 & 49 \\
Proportion of patients retained & $82 \%$ & $82 \%$ & $78 \%$ & $72 \%$ \\
\hline
\end{tabular}

Table 7. PTA use frequency and volume of data input

\begin{tabular}{lc}
\hline App usage frequency during study period & No. of days \\
\hline Average number of days tracked during study period & 48 \\
Maximum number of days tracked & 113 \\
App data input volume during study period & Number \\
Average no. of symptom scores entered in motif interface & 629 \\
Maximum no. of symptom scores entered & 3608 \\
Median no. of symptom scores entered & 565 \\
\hline
\end{tabular}

\begin{tabular}{|lc|}
\hline Table 8. & PTA user retention after trial period (as of March 2016) \\
\hline & No. of participants \\
\hline Tracking for $>182$ days (i.e. $>6$ months) & 18 \\
Tracking for $>365$ days (i.e. $>12$ months) & 3 \\
& Days \\
Longest use recorded & 510 \\
\hline
\end{tabular}

adherence chose participants with lower adherence ${ }^{36}$ as criteria for inclusion in a trial, which translates to an increased potential for improvement. We did not exclude patients with high selfreported adherence, indicating that PTA can help a range of people with varying levels of adherence.

To study the impact of the PTA on health services use, we need to assess the PTA over a longer duration. Although it seems reasonable to conclude that improvement in adherence is desirable, specifics around how much self-reported adherence is clinically meaningful in PD will require additional studies looking specifically at clinician and carer measures.

In summary, we conclude that the PTA could be an effective and novel way of enhancing short-term self-reported medication

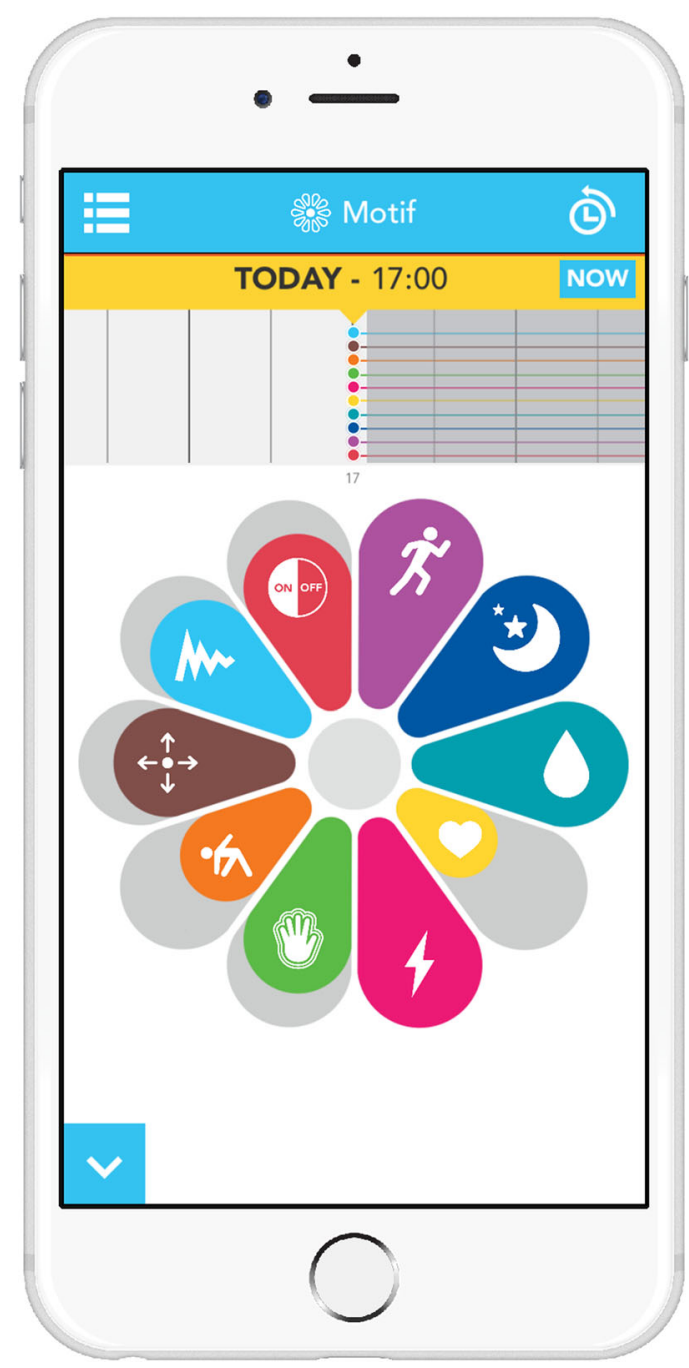

Fig. 3 Screenshot of PTA self-tracking interface

adherence and quality of clinical consultation by supporting selfmanagement in PD.

\section{METHODS}

An open-label, multicentre RCT was conducted from August 2014 to June 2015. The objective was to compare the use of smartphone and internetenabled PTA with TAU for 16 weeks among patients with PD across 7 
centres in England and Scotland (John van Geest Centre for Brain Repair, Cambridge University Hospitals NHS Trust, Cambridge UK; King's College Hospital NHS Foundation Trust, London, UK; Newcastle upon Tyne Hospitals NHS Foundation Trust, Newcastle, UK; NHS Forth Valley, Scotland, UK; St. George's Healthcare NHS Trust, London, UK; The Walton Centre NHS Foundation Trust, Liverpool, UK ; University Hospitals Birmingham NHS Foundation Trust, Birmingham, UK.) Ethics was obtained from the National Research Ethics Service London-Westminster Research Ethics Committee $(13 / L O / 1783)$. Details of the trial are available in the trial protocol. ${ }^{37}$

Eligibility criteria were drawn up in a pragmatic manner to demonstrate both the effectiveness and the ease of implementation of the PTA if used in routine clinical practice.

Inclusion criteria included, (i) a diagnoses of probable, idiopathic PD, (ii) on one or more Parkinson's medications not altered within the previous month and not expected to change during the trial period, and, (iii) English-speaking and literate with access to a smartphone and/or tablet or internet on a daily basis at home. Key exclusion criteria included (i) suspected Parkinsonism due to causes other than idiopathic PD, (ii) current or previous treatment for side effects of prolonged neuroleptic treatment and, (iii) a diagnosis of dementia or significant cognitive impairment or major psychiatric illness associated with psychosis or a major, serious comorbid illness (as recorded in patient case file).

\section{Parkinson's tracker app}

The clinical team identified potentially eligible patients across the seven trial sites from the clinic lists 6 weeks prior to upcoming outpatient (OP) appointments. An information pack containing a patient invitation letter, a participant information sheet and a consent form was sent to potential

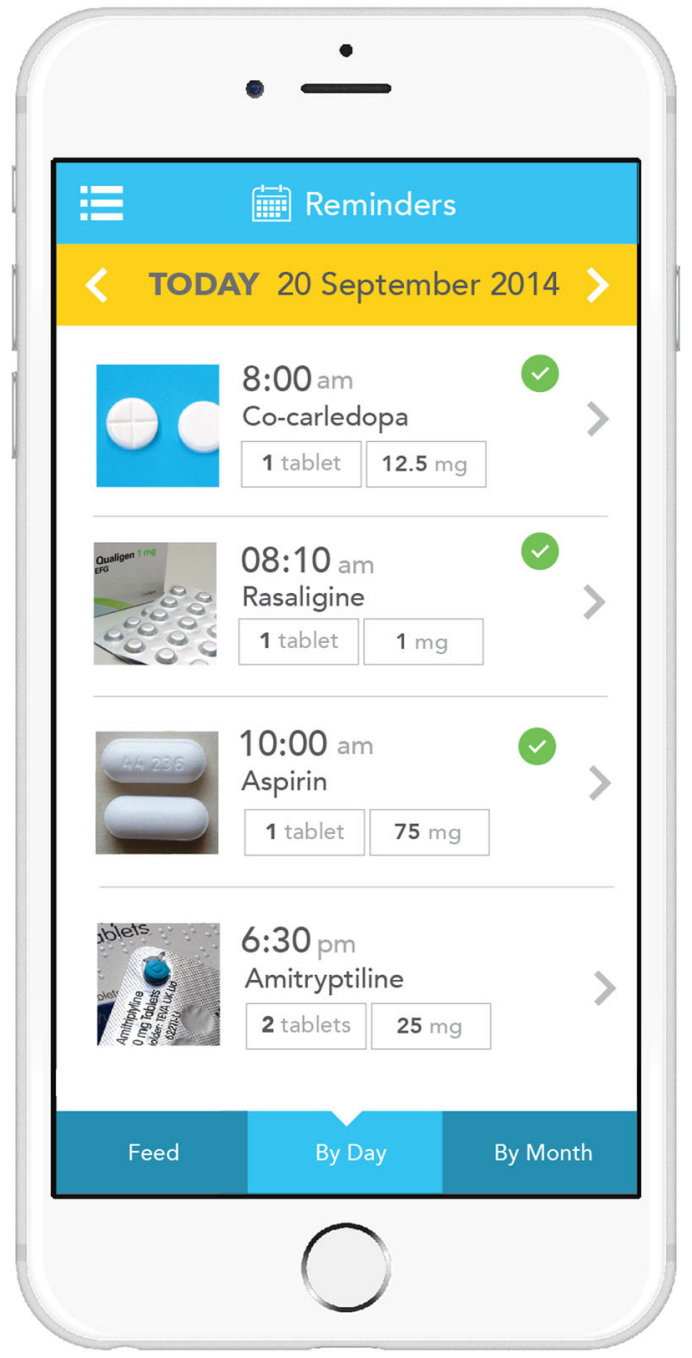

Fig. 4 Screenshot of PTA medication reminders participants 3-4 weeks prior to their next OP clinic appointment. At the OP appointment, the clinician rechecked eligibility criteria and administered the informed consent.

Following consent, patients were randomised to either the PTA or the TAU group. They completed the baseline questionnaires at the clinic or were asked to complete it at home within 1 week of their OP appointment and were given the date for follow-up. Apart from consent forms, no paper-based questionnaires or forms were used.

Patient questionnaires were administered online using a secure research-grade questionnaire tool (Qualtrics) and other data was collected using site specific OpenClinica electronic Research Form.

Those allocated to the PTA arm received instructions from their clinical team to download the PTA to their Android or iPhone smartphones or tablet devices (using the free 'uMotif' app from the Google Play Store or Apple App Store) or to access it via a website portal within 1 day after they have attended their OP appointment.

The app consisted of the following features:

1. A sliding petal interface to track ten self-monitoring measures, chosen by the trial team, on a 5-point scale: including sleep, exercise, mood, energy, movement, suppleness (Fig. 3). An accessibility mode with a zoom function to magnify the screen was included.

2. A reminder system for patients to set up to allow the patient to receive alerts to help track medication (Fig. 4)

3. An option to generate a report of data entered by the patient over the trial period as an aid at their 16 week follow-up appointment (Fig. 5)

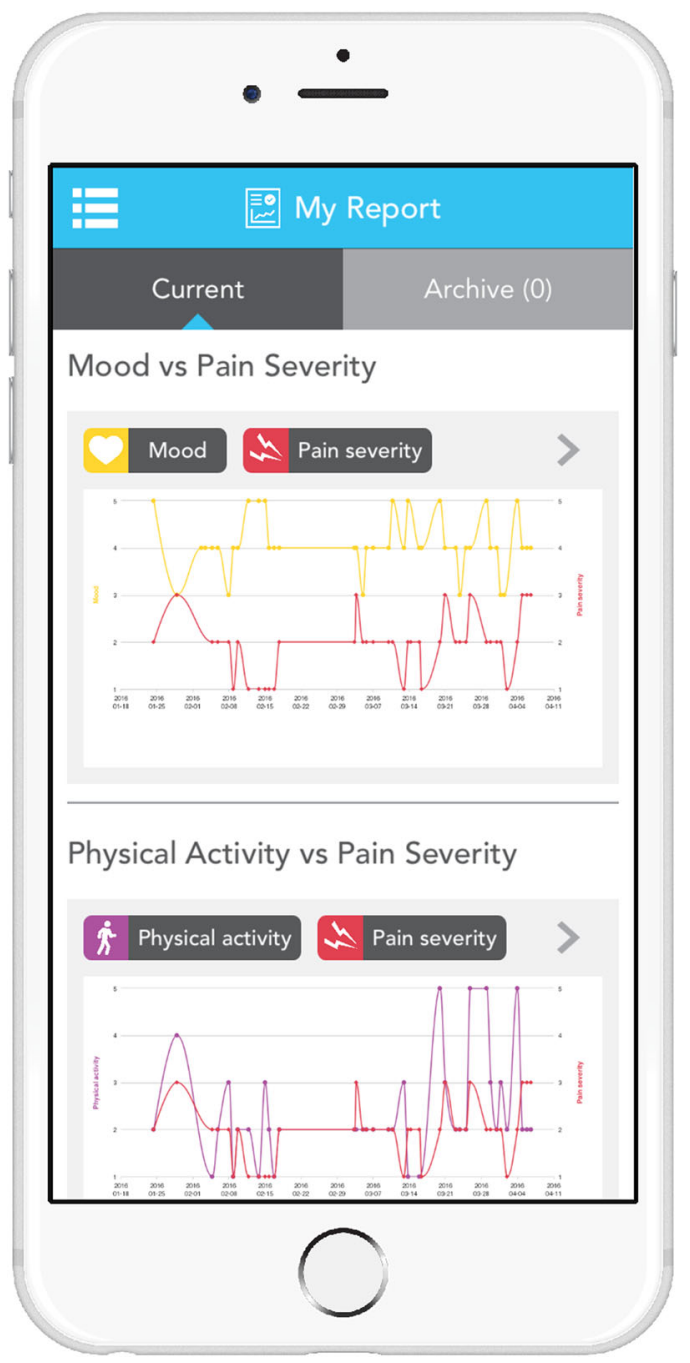

Fig. 5 Screenshot of PTA report 


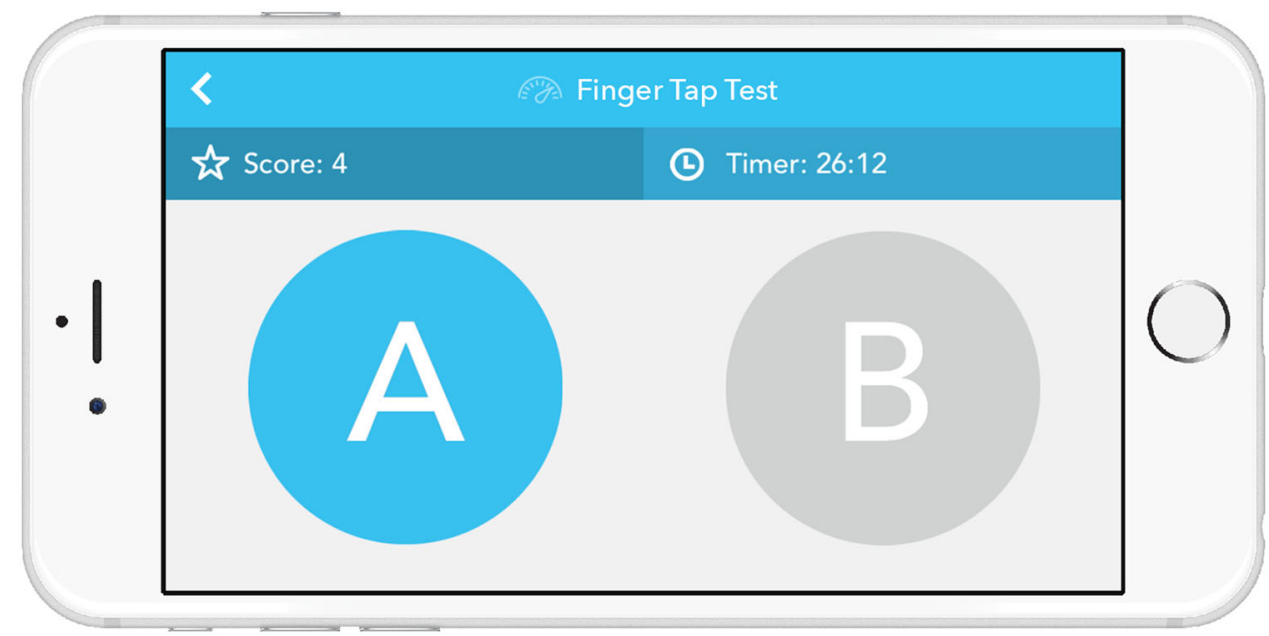

Fig. 6 Screenshot of PTA finger tapping test

4. Games to track physical responsiveness (finger-tapping task) (Fig. 6) and cognition (number-size Stroop test)

5. Information about PD from Parkinson's UK and the Cure Parkinson's Trust

Participants were asked to set up medication reminders and use the app once a day or if not possible at least on alternate days over 16 weeks. They also received a call from their clinician after 2 weeks to check if they, (i) had completed the baseline study questionnaires, (ii) had downloaded the app and, (iii) were having any difficulties with the PTA. At the 16-week visit participants used the data they collected on their app during their consultation. Technical support was available for patients via email throughout the study if they experienced problems with the PTA.

Participants in the TAU group received a call from their clinicians after 2 weeks to check if they had completed the study questionnaires. At the 16 weeks appointment, they had their regular OP clinical assessments, including symptom review followed by a medication review. Participants in the TAU group were offered access and use of the PTA after their 16 weeks of TAU

Participants in both groups received a reminder phone call from their clinician 1 to 2 weeks prior to the follow-up appointment. Participants completed the end of trial questionnaires within 1 week after their 16week follow-up appointment.

Participants in both arms had 2 contacts at the clinic. Visit 1 included talking through the consent, randomisation and giving access to the app if allocated to the intervention. This took between $30-45 \mathrm{~min}$ Visit 2 at the end of the study period included reviewing the data collected in the app for the intervention and for control included inviting to the app and lasted $30 \mathrm{~min}$

Recruitment was originally planned as a rolling programme over the course of 16 weeks until recruitment targets are achieved. However, we extended the recruitment by another 6 months to help recruit the required patient numbers.

\section{Outcome measures}

The primary outcome measure was self-reported adherence to treatment as determined by Morisky Medication Adherence Scale (MMAS-8). ${ }^{38}$ This is a self-report eight-item scale with response categories of 'yes' or 'no' for each item and a 5-point Likert response for the last item. Higher responses indicate better adherence. Secondary outcome measures included:

1. Parkinson's Disease Questionnaire (PDQ-39): ${ }^{39}$ Measures eight dimensions of QoL-mobility, activities of daily living, emotional wellbeing, stigma, social support, cognition, communication and bodily discomfort. Lower total scores reflect better QoL.

2. Patient-Centered Questionnaire for PD: ${ }^{40}$ (PCQ-PD) to measure changes in quality of consultation. PCQ-PD is a self-completed questionnaire addressing forty-four care aspects in six subscales (information, multidisciplinary collaboration, accessibility, empathy, patient involvement and emotional support) of patient-centeredness.
3. Non-Motor Symptom Questionnaire: ${ }^{41}$ A 30-item self-completed questionnaire featuring responses as "yes," or "no" to each item.

4. Hospital Anxiety and Depression Scale: ${ }^{42}$ A self-screening questionnaire for anxiety and depression. It consists of 14 questions with seven each for anxiety and depression.

5. Beliefs about Medication Questionnaire: ${ }^{43}$ A self-administered questionnaire and comprising of two subscales: An 11-item questionnaire relating to prescribed medication and an 8-item questionnaire relating to general views about taking medication. Respondents rate each item on a five point Likert-type scale depending on their degree of agreement (1 strongly disagree, 5 strongly agree). Higher scores indicate higher levels of concern or strong beliefs towards the use of medication.

All measures were collected at baseline and at end of the trial period of 16 weeks.

\section{Sample size}

The sample size calculation was based on the primary endpoint. To detect a 1-point improvement on the MMAS-8 with a standard deviation (SD) of 2.5 and $80 \%$ power at the $5 \%$ significance level would require 200 subjects (100 in each group, 1:1 allocation). To allow for $10 \%$ loss due to dropouts and those lost to follow-up, we aimed to recruit 222 subjects (111 in each group, 1:1 allocation).

\section{Randomisation and masking}

Eligible patients were allocated in a 1:1 ratio to the two arms of the study according to a computer-generated random sequence stratified by centre and using blocks of variable size. The allocation sequence was generated by an independent statistician and was not available to any member of the research team until databases were completed and locked. Copies of the allocation sequence were not held at the recruiting centres. Once randomised, clinicians who enroled participants informed them of their assignment. Blinding to group allocation was not possible, as participants knew whether or not they were receiving PTA. The trial statisticians were blinded to the treatment code when developing the statistical analysis plan and writing the statistical programmes, which were validated and completed using dummy randomisation codes. The actual allocation code was only provided to the trial statisticians after locking of the database and finalisation of the statistical analysis plan.

\section{Statistical methods}

A Generalised Linear Model (GLM) was used for analysis of the primary endpoint and had treatment as the fixed effect and baseline measurement of the primary endpoint as the covariate. The treatment difference together with its $95 \%$ confidence interval between PTA and TAU in the least square mean of primary endpoint was derived from the GLM model. Model assumptions about residuals in regression analysis were checked by inspection of residuals vs. a fitted values plot and no serious violation of 
normality assumption was found. In addition, adjusted analysis and subgroup analysis with pre-specified covariates (age, gender and number of co-morbidities) were performed. For the secondary outcomes (QoL, depression, anxiety, non-motor symptoms, and degrees of depression and anxiety and quality of consultation), the analyses were performed in an analogous fashion within the framework of GLM. Analyses of the primary and secondary outcomes were carried out in adherence to the intention-to-treat principle. In addition, supplemental per-protocol analyses were performed.

\section{CHANGE HISTORY}

A correction to this article has been published and is linked from the HTML version of this article.

\section{ACKNOWLEDGEMENTS}

We are grateful to Oxford University Innovations' Clinical Outcomes for supporting the trial for use of the PDQ-39. We thank the following team members for helping us run the trial: David Thomson at Forth Valley NHS Trust, Louise Fasting and Helen Leggett at the Walton Centre NHS Foundation Trust, Dhaval Trivedi, Lauren Perkins and Rona Inniss at Kings Kings College Hospital NHS Foundation Trust, Alison Leake at St George's NHS Foundation Trust, Philip Brown and Leanne Thompson at Newcastle Upon Tyne NHS Foundation Trust and, Sarah Dhariwal and Claire Arthur at University Hospitals Birmingham NHS Foundation Trust. Our special thanks to the Cure Parkinson's Trust and Parkinson's UK for providing content for the PTA and for being part of the Trial Management Group. Other information. This work was commissioned by NHS Midlands and East and funded by the Department of Health.

\section{AUTHOR CONTRIBUTIONS}

R.L. and B.H. conceived the study and, in collaboration with D.B., R.C., C.G., S.P., R.S., J. S., L.R., RB., M.S., J.T., D.W. and A.W. wrote and refined the protocol; D.B., R.C., C.G., S.P., N.G., S.S., M.S., J.T., R.B., L.R., A.W. led recruitment at sites. B.B. and M.E. helped with the analysis and interpretation of the PCQ-PD results. B.J. was chief designer of the software platform. D.W. calculated the sample size. D.W. and E.W. designed the data analysis plan and completed the statistical analysis. All authors read and approved the final manuscript.

\section{COMPETING INTERESTS}

R.L., B.H. and B.J. are employees of uMotif. uMotif has other projects involved with delivering smartphone solutions for chronic disease management. Authors not associated with uMotif declare no conflict of interest.

\section{REFERENCES}

1. Malek, N. \& Grosset, D. G. Medication adherence in patients with Parkinson's disease. CNS Drugs 29, 47-53 (2015)

2. European Parkinson's Disease Association (EPDA) What is Parkinson's? http://www.epda.eu.com/en/pd-info/about-parkinsons/

3. Van Den Eeden, S. K. et al. Incidence of Parkinson's disease: variation by age, gender, and race/ethnicity. Am. J. Epidemiol. 157, 1015-1022 (2003).

4. Shulman, L. M., Taback, R. L., Rabinstein, A. A. \& Weiner, W. J. Non-recognition of depression and other non-motor symptoms in Parkinson's disease. Parkinsonism. Relat. Disord. 8, 193-197 (2002).

5. Todorova, A., Jenner, P., Chaudhuri, K. R. Non-motor Parkinson's: integral to motor Parkinson's, yet often neglected. Pract. Neurol. 14, 310-322 (2014).

6. Pressley, J. C. et al. The impact of comorbid disease and injuries on resource use and expenditures in Parkinsonism. Neurology. 60, 87-93 (2003).

7. National Institute for Health and Care Excellence (NICE): Parkinson's disease in over 20s: diagnosis and management. https://www.nice.org.uk/guidance/cg35/ resources/parkinsons-disease-in-over-20s-diagnosis-and-management-97538823 7765 (2006)

8. Schapira, A. H. et al. Perspectives on recent advances in the understanding and treatment of Parkinson's disease. Eur. J. Neurol. 16, 1090-1099 (2009).

9. Tan, E. K., Yeo, A. P., Tan, V., Pavanni, R. \& Wong, M. C. Prescribing pattern in Parkinson's disease: are cost and efficacy overriding factors?. Int. J. Clin. Pract. 59, 511-514 (2005)

10. Institute of Medicine (US). Committee on Quality of Health Care in America. Crossing the quality chasm: a new health system for the 21st century. National Academy Press. https://www.nationalacademies.org/hmd/ /media/Files/Report \%20Files/2001/Crossing-the-Quality-Chasm/Quality\%20Chasm\%202001\%20\%20 report\%20brief.pdf (2001).
11. van der Eijk, M., Faber, M. J., Al Shamma, S., Munneke, M. \& Bloem, B. R. Moving towards patient-centered healthcare for patients with Parkinson's disease. Parkinsonism. Relat. Disord. 17, 360-364 (2011).

12. Kralik, D., Koch, T., Price, K. \& Howard, N. Chronic illness self-management: taking action to create order. J. Clin. Nurs. 13, 259-267 (2004).

13. Dayer, L., Heldenbrand, S., Anderson, P., Gubbins, P. O. \& Martin, B. C. Smartphone medication adherence apps: potential benefits to patients and providers. J. Am. Pharm. Assoc. 53, 172-181 (2013).

14. Wroe, A. L. Intentional and unintentional nonadherence: a study of decision making. J. Behav. Med. 25, 355-372 (2002).

15. luga, A. O. \& McGuire, M. J. Adherence and health care costs. Risk Manag Healthc Policy 7, 35 (2014).

16. Elliott, R. A., et al. Department of Health Policy Research Programme Project "Understanding and Appraising the New Medicines Service in the NHS in England (029/0124)." 2014. 1-20 (Nottingham University School of Pharmacy, 2014).

17. Psaty, B. M., Koepsell, T. D., Wagner, E. H., LoGerfo, J. P. \& Inui, T. S. The relative risk of incident coronary heart disease associated with recently stopping the use of ß-blockers. JAMA 263, 1653-1657 (1990).

18. Lau, D. T. \& Nau, D. P. Oral antihyperglycemic medication nonadherence and subsequent hospitalization among individuals with type 2 diabetes. Diabetes Care 27, 2149-2153 (2004).

19. Krishna, S., Boren, S. A. \& Balas, E. A. Healthcare via cell phones: a systematic review. Telemed. e-Health 15, 231-240 (2009).

20. Narayana, R., Hellman,B., Addyman, C., Stamford,J. Self management in long term conditions using smartphones: a pilot study in Parkinson's disease. (Conference abstract). www.ijic.org/articles/1820/galley/2648/download/ (2014).

21. Morisky, D. E., Ang, A., Krousel-Wood, M., Ward, H. Predictive validity of a medication adherence measure for hypertension control. J. Clin. Hypertens. 10, 348-354 (2008).

22. Krousel-Wood, M. A. et al. New medication adherence scale versus pharmacy fill rates in seniors with hypertension. Am. J. Manag. Care 15, 59-66 (2009).

23. Morisky, D. E., DiMatteo, M. R. Improving the measurement of self-reported medication nonadherence: final response. J Clin Epidemio 64, 258-263 (2011). PMID:21144706.

24. Schapira, A. H. V., Emre, M., Jenner, P. \& Poewe, W. Levodopa in the treatment of Parkinson's disease. Eur. J. Neurol. 16, 982-989 (2009).

25. Fleisher, J. E. \& Stern, M. B. Medication non-adherence in Parkinson's disease. Curr. Neurol. Neurosci. Rep. 13, 382 (2013).

26. Dubois, B. \& Pillon, B. Cognitive deficits in Parkinson's disease. J. Neurol. 244, 2-8 (1996)

27. Daley, D. J., Myint, P. K., Gray, R. J. \& Deane, K. H. Systematic review on factors associated with medication non-adherence in Parkinson's disease. Parkinsonism Relat. Disord. 18, 1053-1061 (2012).

28. Patel, K. Optimising medication for Parkinson's disease patients with dysphagia Br. J. Community Nurs. 20, 322, 324-326 (2015).

29. Grosset, K. A. \& Grosset, D. G. Patient-perceived involvement and satisfaction in Parkinson's disease: Effect on therapy decisions and quality of life. Mov. Disord. 20, 616-619 (2005).

30. Chen, A. Mobile retention benchmarks for 2014 vs 2013 show a 50\% drop in D1 retention. http://andrewchen.co/mobile-retention-benchmarks-for-2014-vs-2013show-a-50-drop-in-d1-retention-guest-post/.

31. Ofcom, Adults' media use and attitudes, 2015.

32. Net Market Share. Search results for operating system share for mobile/tablet users between August 2014-June 2015. https://www.netmarketshare.com/ operating-system-market-share.aspx?qprid=8\&qpcustomd=1\&qpsp=187\&qpnp= 11\&qptimeframe $=M$.

33. Treweek, S. et al. Methods to improve recruitment to randomised controlled trials: Cochrane systematic review and meta-analysis. BMJ Open 3, e002360 (2013).

34. Elm, J. J. et al. Self-reported adherence versus pill count in Parkinson's disease: The NET-PD experience. Mov. Disord. 22, 822-827 (2007).

35. Muntner, P. et al. Defining the minimal detectable change in scores on the eightitem Morisky Medication Adherence Scale. Ann. Pharmacother. 45, 569-575 (2011).

36. Daley, D. J. et al. Adherence therapy improves medication adherence and quality of life in people with Parkinson's disease: a randomised controlled trial. Int. J. Clin. Pract. 68, 963-971 (2014).

37. Lakshminarayana, R. et al. Smartphone-and internet-assisted self-management and adherence tools to manage Parkinson's disease (SMART-PD): study protocol for a randomised controlled trial (v7; 15 August 2014). Trials. 15, 374 (2014).

38. Morisky, D. E., Green, L. W. \& Levine, D. M. Concurrent and predictive validity of a self-reported measure of medication adherence. Med. Care 24, 67-74 (1986). 
39. Peto, V., Jenkinson, C., Fitzpatrick, R. \& Greenhall, R. The development and validation of a short measure of functioning and well being for individuals with Parkinson's disease. Qual. Life Res. 4, 241-248 (1995).

40. van der Eijk, M. et al. Patient-centeredness in PD care: development and validation of a patient experience questionnaire. Parkinsonism Relat. Disord. 18, 1011-1016 (2012)

41. Chaudhuri, K. R. et al. 'An international multicentre pilot study of the the first comprehensive self-completed non motor symptoms questionnaire for Parkinson's disease: The NMSQuest study'. Mov. Disord. 21, 916-923 (2006).

42. Zigmond, A. S. \& Snaith, R. P. The hospital anxiety and depression scale. Acta Psychiatr. Scand. 67, 361-370 (1983).

43. Horne, R., Weinman, J. \& Hankins, M. The beliefs about medicines questionnaire: the development and evaluation of a new method for assessing the cognitive representation of medication. Psychol. Health 14, 1-24 (1999).

(c) (i)

This work is licensed under a Creative Commons Attribution 4.0 International License. The images or other third party material in this article are included in the article's Creative Commons license, unless indicated otherwise in the credit line; if the material is not included under the Creative Commons license, users will need to obtain permission from the license holder to reproduce the material. To view a copy of this license, visit http://creativecommons.org/licenses/by/ $4.0 /$

(c) The Author(s) 2017

Supplementary Information accompanies the paper on the npj Parkinson's Disease website (doi:10.1038/s41531-016-0003-z). 